\title{
SLC26A4 c.919-2A>G varies among Chinese ethnic groups as a cause of hearing loss
}

Pu Dai, MD, $P h D^{1}$, Qi Li, MD ${ }^{1}$, Deliang Huang, $M D$, PhD ${ }^{1}$, Yongyi Yuan, $M D$, $P h D^{1}$, Dongyang Kang ${ }^{1}$, David T. Miller, $M D$, $P h D^{2}$, Hong Shao, $B A^{2}$, Qingwen $Z h u, M D, P h D^{1}$, Jia He, $M D, P h D^{3}, F e i Y u, M D, P h D^{1}$, Xin Liu, $M D, P h D^{1}$, Bing Han, $M D^{1}$, Huijun Yuan, $M D$, PhD ${ }^{1}$, Orah S. Platt, $M D^{2}$, Dongyi Han, $M D, P h D^{1}$, and Bai-Lin Wu, MMed, $P h D^{2}$

\begin{abstract}
Purpose: Mutations in the SLC26A4 gene are second only to GJB2 mutations as a currently identifiable genetic cause of sensorineural hearing loss. In most areas of China, genetic testing for sensorineural hearing loss is unavailable because of limited knowledge of the mutation spectrum. Although SLC26A4 c.919-2A>G (IVS7-2A>G) is a common mutation among some Asian populations, the mutation prevalence among various ethnic groups within China has not been studied. Methods: DNA specimens from 3271 subjects with moderate to profound sensorineural hearing loss from 27 regions of China were genotyped for the c.919-2A $>$ G mutation by polymerase chain reaction/restriction-fragment-length polymorphism. Normal hearing controls from Han $(n=185)$ and Uigur ( $n=152)$ populations were also tested. Results: Overall, 408 subjects with sensorineural hearing loss (12.5\%) carried at least one c.919-2A $>$ G allele, with $158(4.8 \%)$ homozygotes and $250(7.6 \%)$ heterozygotes. Within the subpopulations examined, the rate varies from $0 \%$ to $12.2 \%$ for c.919-2A $>$ G homozygotes and from $0 \%$ to $17.6 \%$ for heterozygotes. Based on this cohort, Chinese subjects with sensorineural hearing loss seem to have a relatively higher c.919-2A $>$ G frequency than that of other Asian populations. Conclusion: These results demonstrate that a simple and efficient genetic test for the c.919-2A $>$ G mutation alone would identify the molecular cause in up to $8-12 \%$ of individuals with sensorineural hearing loss in a few eastern and central regions of China. Those who are negative for the c.919-2A $>$ G mutation would be candidates for further mutational analysis of SLC26A4 or other deafness-related genes. This would greatly improve genetic diagnosis and counseling for a huge number of Chinese individuals and family members with sensorineural hearing loss in China, and many more ethnic Chinese in other countries, which might be up to one million. Genet Med 2008:10(8):586-592.
\end{abstract}

Key Words: SLC26A4, c.919-2A>G (IVS7-2A>G), hearing loss, SNHL, deafness, Chinese, genetic epidemiology, DNA diagnostics

Sensorineural hearing loss (SNHL) is the most common neurosensory disorder in humans with an incidence of approximately 1 in 1000 children. Hereditary SNHL is genetically heterogeneous, and causative mutations are identified in approximately $50-60 \%$ of cases. ${ }^{1}$ Nonsyndromic deafness accounts for $60-70 \%$ of inherited SNHL and involves more than

From the ${ }^{I}$ Department of Otolaryngology and Genetic Testing Center for Deafness, Chinese PLA General Hospital, Beijing, People's Republic of China; ${ }^{2}$ Division of Genetics, Departments of Laboratory Medicine and Pathology, Children's Hospital Boston, Harvard Medical School, Boston, Massachusetts; and ${ }^{3}$ Department of Health Statistics, Second Military Medical University, Shanghai, People's Republic of China.

Bai-Lin Wu, MMed, PhD, Department of Laboratory Medicine, Children's Hospital Boston, 300 Longwood Avenue, Boston, MA 02115. E-mail: bai-lin.wu@childrens.harvard.edu.

Pu Dai, Qi Li, and Deliang Huang contributed equally to this article.

Dongyi Han is senior author for the group of Chinese PLA General Hospital; Bai-Lin Wu is senior author for Children's Hospital, Boston team.

Disclosure: The authors declare no conflict of interest.

Submitted for publication December 12, 2007.

Accepted for publication April 18, 2008.

DOI: 10.1097/GIM.0b013e31817d2ef1
100 different genes demonstrating autosomal dominant (DFNA), autosomal recessive (DFNB), X-linked (DFN), and maternal inheritance. ${ }^{2}$

Mutations in SLC26A4 cause autosomal recessive SNHL, either in combination with goiter as a component of Pendred syndrome (PS; OMIM no. 274600) or as nonsyndromic hearing loss without goiter (DFNB4; OMIM no. 600791). ${ }^{3-5}$ DFNB4 and PS have been estimated to account for $4-10 \%$ of all hereditary deafness. ${ }^{6}$

SNHL in both DFNB4 and PS is frequently associated with temporal bone abnormalities, including cochlear malformations ranging from isolated enlargement of the vestibular aqueduct (EVA) to Mondini dysplasia where EVA is combined with an incomplete number of turns in the cohlea. EVA can be detected by computerized tomography (CT) or magnetic resonance imaging, 7,8 and bilateral EVA is the most frequent form of temporal bone abnormalities. ${ }^{5}$

A recent study found EVA in approximately $16 \%$ of subjects with idiopathic SNHL, ${ }^{9}$ making it second only to GJB2 (connexin 26) mutations as a cause of autosomal recessive SNHL. 
Among over 150 SLC26A4 mutations, reported either in the online database ${ }^{10}$ or described in the literature, most have been identified in western populations with European ancestry and most are private mutations seen in only single families. ${ }^{3-5,10-21}$ Among Asian populations, EVA associated with a distinctive SLC26A4 mutation spectrum with highly prevalent founder mutations has been observed, such as p.H723R in Japan and Korea and c.919-2A $>G$ (often referred to as IVS7-2A $>G$ ) in Taiwan and mainland China. ${ }^{5,17-19}$

The c.919-2A $>$ G mutation was first identified in a Turkish family ${ }^{22}$ and subsequently detected in deaf subjects from Japan, Korea, and China. ${ }^{15-18}$ c.919-2A $>$ G has not been found in western populations, including deaf individuals living in the United States. ${ }^{10-12,15,16,23}$ One study showed evidence that the c.919-2A $>$ G (IVS7-2A $>$ G) mutation arose from a common ancestor. ${ }^{24}$ The report also demonstrated that this mutation results in skipping of exon 8 with a resulting stop codon at position 311, resulting in a predicted truncated protein. ${ }^{24}$

An estimated 30,000 babies are born with congenital SNHL each year in China, ${ }^{25}$ but many are not offered genetic testing because of limited resources and because few common genetic variants have been identified in Chinese deaf children. China is a large and diverse country accounting for nearly one quarter of the world's population and representing 56 ethnic groups (e.g., Han, Man, Mon, Hui, Zang, Miao, Yi, Wa, Bai, Zhuang, Wei, Qiang, and $\mathrm{NaXi}$ ) clustered in different parts of the country. Most of the 1.3 billion Chinese (91.9\%) are Han from the middle and eastern parts of China (http://education. yahoo.com/reference/factbook/ch/popula.html; accessed August 14, 2007). Little is known about the prevalence and ethnic distribution of SLC26A4 mutations among individuals with Chinese ancestry. In an effort to provide better knowledge about mutation prevalence that will translate into improved utilization of limited resources for genetic testing for SNHL, we report the population prevalence for a common SLC26A4 mutation, c.919-2A $>$ G, among 3271 subjects with moderate to profound SNHL from 27 regions of China. This is essentially a complete geographic coverage of China to have at least a representative region from each area, which also include the regions that have more ethnic groups.

\section{MATERIALS AND METHODS}

\section{Deaf subjects and control samples}

A total of 3271 unrelated students (1879 males and 1392 females; age range, $4-20$ years; mean \pm SD, $13.84 \pm 5.08$ years) with moderate to profound SNHL were recruited from 27 regions of China. All the subjects are probands. No parents or siblings were included in this study. The majority of subjects (2801 of 3271; 85\%), were Han Chinese. Tables 1 and 2 summarize the distribution of subjects by regions and ethnic groups. Eastern and southeast coastal provinces are predominantly Han (90-98\%). The remote northwestern provinces of Xinjiang (New Territory), Xizhan (Tibet), and Qinghai are predominantly ethnic minorities. Individuals from the southwestern provinces of Yunnan and Guizhou, often come from one of several ethnic groups originating from various native tribes. Subjects were identified through the local school systems to ensure that they would be reasonably representative of the general population for geographic regions and ethnic groups.

Informed consent was obtained from all subjects, or from parents of subjects younger than age 18, according to a protocol approved by the ethics committee of the Chinese PLA General Hospital. Ethnicity was categorized according to the information recorded in each individual's permanent residency documentation. Parents were interviewed to obtain family history and a maternal pregnancy history in addition to the subjects' age at diagnosis and clinical history, including infections, possible head or brain injuries, and exposure to aminoglycoside antibiotics. Only subjects with SNHL were included in this study. These subjects showed moderate to profound bilateral SNHL on audiograms. In addition, subjects with mental retardation, blindness and other severe systemic problem were excluded from this study. Goiter is not an exclusion criterion, although we did not find any case with goiter among the 3271 subjects in this cohort.

We analyzed 337 control individuals with normal hearing using the same screening method. Among controls, 185 were Han Chinese from two densely populated regions, Beijing City (northern region) and Jiangsu Province (eastern region), and 152 were from Uigur City of Xinjiang, the most northwestern remote region of China, as a non-Han control for comparison; because the genetic background of Uigur population is significantly different from the Han population.

\section{DNA samples and genotyping}

All the samples were processed at the Chinese PLA General Hospital. DNA was extracted from peripheral blood using a commercially available kit (Watson Biotechnologies Inc., Shanghai, China). Genotyping for c.919-2A $>\mathrm{G}$ was performed by polymerase chain reaction (PCR) with modified primers (Forward 5' $5^{\prime}$ TGGAGTTTTTAACATCTTTTGTTTTATTCC-3'; Reverse 5' -CCCTTGGGATGGATTTAACA-3') to produce a $114 \mathrm{bp}$ product. The forward primer was designed to introduce an HpaII site for the c.919-2A>G minor allele only. Four microliters of each PCR product was analyzed by electrophoresis on a $2.0 \%$ agarose gel, and $5 \mu \mathrm{L}$ was digested by HpaII in a $20 \mu \mathrm{L}$ reaction at $37^{\circ} \mathrm{C}$ for 2 hours. Digestion products were analyzed by electrophoresis through an $8.0 \%$ polyacrylamide gel followed by silver staining to identify the $114 \mathrm{bp}$ band from the major allele or an $85 \mathrm{bp}$ band from the minor allele, or both in the case of a heterozygote. A 29 bp band produced from digestion of PCR product bearing the minor allele was not detected reliably on the gel (Fig. 1).

Total of 408 positive samples and 1350 randomly selected negative samples were confirmed by direct sequencing using an ABI 3100 sequencer. No false positives or false negatives were found in this parallel study. All the genotyping and sequencing were performed at the Chinese PLA General Hospital. 
Table 1

Prevalence of SLC26A4 c.919-2A > G homozygote, heterozygote, and allele frequency in China by regions

\begin{tabular}{|c|c|c|c|c|c|c|c|}
\hline City, province & $\begin{array}{l}\text { Population } \\
\text { size }(\mathrm{M})^{a}\end{array}$ & $\begin{array}{l}\text { Han } \\
(\%)^{b}\end{array}$ & $\begin{array}{c}\text { Subjects } \\
\text { with SNHL }\end{array}$ & $\begin{array}{c}\text { Subjects with } \\
\text { c.919-2A>G, } n(\%)\end{array}$ & $\begin{array}{c}\text { Homozygotes, } \\
n(\%)\end{array}$ & $\begin{array}{c}\text { Heterozygotes, } \\
n(\%)\end{array}$ & $\begin{array}{c}\text { Allele } \\
\text { frequency }\end{array}$ \\
\hline Anyang, Henan & 5.3 & 98.7 & 158 & $44(27.8)$ & $19(12.2)$ & $25(15.8)$ & 0.199 \\
\hline $\begin{array}{l}\text { Zhuozhou-Gaobeidian, } \\
\text { Hebei }\end{array}$ & 1.2 & 95.6 & 68 & $18(26.5)$ & $6(8.8)$ & $12(17.6)$ & 0.176 \\
\hline Xi'an, Shaanxi & 7.3 & 99.5 & 56 & $11(19.6)$ & $6(10.7)$ & $5(8.9)$ & 0.152 \\
\hline Wuhan, Hubei & 7.9 & 95.6 & 87 & $18(20.7)$ & $7(8.0)$ & $11(12.6)$ & 0.144 \\
\hline Shanghai & 13.5 & 99.4 & 71 & $14(19.7)$ & $5(7.0)$ & $9(12.7)$ & 0.134 \\
\hline Datong, Shanxi & 3.0 & 99.9 & 174 & $34(19.5)$ & $9(5.2)$ & $25(14.4)$ & 0.124 \\
\hline $\begin{array}{l}\text { Mudanjiang, } \\
\text { Heilongjiang }\end{array}$ & 2.7 & 95.1 & 37 & $5(13.5)$ & $4(10.8)$ & $1(2.7)$ & 0.122 \\
\hline Lanzhou, Gansu & 3.1 & 91.2 & 56 & $11(19.6)$ & $2(3.6)$ & $9(16.1)$ & 0.116 \\
\hline Chifeng, Inner Mongol & 4.5 & 79.2 & 128 & $18(14.1)$ & $9(7.0)$ & $9(7.0)$ & 0.105 \\
\hline Jilin, Jilin & 4.3 & 90.8 & 57 & $8(14.0)$ & $3(5.3)$ & $5(8.8)$ & 0.096 \\
\hline Foshan, Guangdong & 3.5 & 98.5 & 183 & $25(13.7)$ & $9(4.9)$ & $16(8.7)$ & 0.093 \\
\hline Beijing & 11.8 & 95.7 & 189 & $26(13.5)$ & $8(4.1)$ & $18(9.3)$ & 0.088 \\
\hline Chengdu, Sichuan & 10.6 & 95.0 & 109 & $14(12.8)$ & $5(4.6)$ & $9(8.3)$ & 0.087 \\
\hline Yuncheng, Shanxi & 4.9 & 99.9 & 216 & $27(12.5)$ & $10(4.6)$ & $17(7.9)$ & 0.086 \\
\hline Nantong, Jiangsu & 7.7 & 99.6 & 195 & $23(11.8)$ & $9(4.6)$ & $14(7.2)$ & 0.082 \\
\hline Fuyang, Anhui & 9.0 & 99.3 & 116 & $14(12.1)$ & $5(4.3)$ & $9(7.8)$ & 0.082 \\
\hline Kunming, Yunnan & 5.0 & 87.4 & 159 & $17(10.7)$ & $9(5.7)$ & $8(5.0)$ & 0.082 \\
\hline Yinchuan, Ningxia & 1.3 & 65.4 & 197 & $25(12.7)$ & $7(3.6)$ & $18(9.1)$ & 0.081 \\
\hline Urumqi, Xinjiang & 1.9 & 75.4 & 191 & $17(8.9)$ & $9(4.7)$ & $8(4.2)$ & 0.068 \\
\hline Korla, Xinjiang & 0.4 & 69.9 & 120 & $11(9.2)$ & $5(4.2)$ & $6(5.0)$ & 0.067 \\
\hline Xining, Qinghai & 1.8 & 54.0 & 67 & $5(7.5)$ & $2(3.0)$ & $3(4.5)$ & 0.052 \\
\hline Binzhou, Shandong & 3.6 & 99.3 & 75 & $4(5.3)$ & $3(4.0)$ & $1(1.3)$ & 0.047 \\
\hline Guiyang, Guizhou & 3.5 & 62.2 & 138 & $8(5.8)$ & $3(2.2)$ & $5(3.6)$ & 0.040 \\
\hline Fuzhou, Fujian & 6.1 & 98.3 & 147 & $8(5.4)$ & $3(2.0)$ & $5(3.4)$ & 0.037 \\
\hline Liuzhou, Guangxi & 3.5 & 61.6 & 94 & $3(3.2)$ & 0 & $3(3.2)$ & 0.016 \\
\hline Lincang, Yunnan & 2.2 & 62.1 & 73 & $1(1.4)$ & $1(1.4)$ & 0 & 0.014 \\
\hline Lhasa, Tibet & 0.4 & 6.1 & 112 & $1(0.9)$ & 0 & $1(0.9)$ & 0.004 \\
\hline Total & 130 & & 3271 & $408(12.5)$ & $158(4.8)$ & $250(7.6)$ & 0.087 \\
\hline
\end{tabular}

In regions of Anyang, Gaobeidian-Zhuozhou, Xi'an, Wuhan, Shanghai, Datong, Mudanjiang, Lanzhou, Jilin, Foshan, Beijing, Yuncheng, Chengdu, Nantong, Fuyang, Binzhou, and Fuzhou City, Han people constitute more than $90 \%$ of the population. Minorities constitute $20.8 \%$ of the population in Chifeng (most are Mon), 30.1\% in Korla (most are Uigur), 24.6\% in Urumqi (most are Uigur), 34.6\% in Yinchuan (most are Hui), 46.0\% in Xining (most are Hui and Zang), and 93.9\% in Lhasa (most are Zang). In the southwestern region of China, minorities constitute $36.4 \%$ of the population in Liuzhou, $37.8 \%$ in Guiyang, $12.6 \%$ in Kunming, and $37.9 \%$ in Lincang. Most minorities originated from various native tribes.

${ }^{a}$ Population size refers to the overall census number of each region $(\mathrm{M}=$ millions) (http://www.xzqh.org/quhua/index.htm).

${ }^{b}$ Percentage of Han people in the population of each region (http://www.cdjsw.gov.cn/Article_Show.asp?ArticleID=462).

\section{Statistical analysis}

Statistical analysis was performed using SAS v9.1.3 software (SAS Inc., Cary, NC). Comparison of c.919-2A $>$ G minor allele frequency (MAF) across all ethnic groups yielded a statistically significant difference. All pairwise comparisons between ethnic groups reflect a corrected significance level: $\alpha^{\prime} \approx 0.00313$ $\left\{\alpha^{\prime}=\alpha /[0.5 k(k-1)+1]=0.05 /[0.5 \times 6 \times(6-1)+1]=\right.$ $0.00313, k$ is the number of group, $\alpha=0.05\}$.

\section{RESULTS}

\section{Geographic distribution of subjects with moderate to profound SNHL in China}

In China, approximately $94 \%$ of the population is concentrated in the southern (e.g., Guangdoung and Fujian provinces) and the eastern (e.g., Beijing, Shanghai, Nanjing) regions (http://www.gxu.edu.cn/administration/gxdxjsb/zzcl/ 
Table 2

High variability of SLC26A4 c.919-2A $>$ G allele frequency in China by ethnic groups

\begin{tabular}{|c|c|c|c|c|c|}
\hline Ethnicity & $\begin{array}{c}\text { Subjects } \\
\text { with SNHL }\end{array}$ & $\begin{array}{c}\text { Subjects with } \\
\text { c.919-2A }>\text { G, } n(\%)\end{array}$ & $\begin{array}{c}\text { Homozygotes, } \\
n(\%)\end{array}$ & $\begin{array}{c}\text { Heterozygotes, } \\
n(\%)\end{array}$ & $\begin{array}{c}\text { Allele } \\
\text { frequency }\end{array}$ \\
\hline Mon & 32 & $5(15.6)$ & $3(9.4)$ & $2(6.3)$ & 0.125 \\
\hline Han & 2783 & $386(13.8)$ & $147(5.3)$ & $239(8.5)$ & 0.096 \\
\hline Hui & 121 & $11(9.1)$ & $5(4.1)$ & $6(5.0)$ & 0.066 \\
\hline Southwest ethnic group & 131 & $4(3.1)$ & $1(0.8)$ & $3(2.3)$ & 0.019 \\
\hline Uigur & 65 & 0 & 0 & 0 & 0.000 \\
\hline Tibetan & 116 & 0 & 0 & 0 & 0.000 \\
\hline
\end{tabular}

Ethnic groups from the northern regions (Man, Korea, Uzbek, and Hazakh) are not included because the total number of samples was small (less than 15).

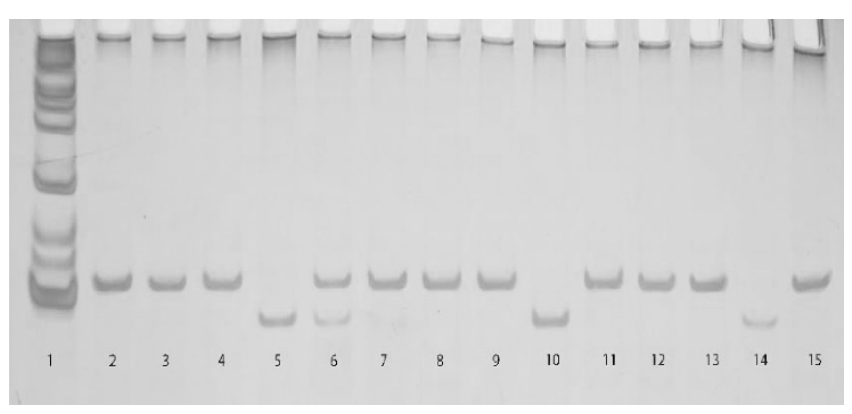

Fig. 1. Polymerase chain reaction/restriction-fragment-length polymorphism analysis of c.919-2A $>$ G mutation. The polymerase chain reaction followed by restriction-fragment-length polymorphism with PSDM and HpaII digestion are described in "Materials and Method." Lane 1 is the DNA marker of 2000, 1000, 750, 500, 250, and $100 \mathrm{bp}$. Lanes 2-13 are the subjects with SNHL genotyped for the c.919-2G $>$ A mutation. Lane 14 is a positive control (homozygous c.919-2A $>\mathrm{G}$ ), which produces bands of 114,86 , and $28 \mathrm{bp}$; but the $28 \mathrm{bp}$ band is not shown well on the gel. Lane 15 is a normal control (wild type, which produces a $114 \mathrm{bp}$ band only). Subjects in lanes 5 and 10 were homozygous for the c.919-2A $>$ G minor allele, the subject in lane 6 was heterozygous, and the remaining lanes represent subjects with no c.919-2A $>$ G minor allele.

rkfb.htm; accessed August 14, 2007). The western half of China is mostly sparsely populated desert (e.g., Xinjiang) and elevated plateau (e.g., Qinghai, Tibet). We studied subjects with moderate to profound SNHL from 27 regions of 24 provinces, including complete geographic coverage of China: one northern province (Inner Mongol), two northeastern provinces (Heilongjiang and Jilin), five eastern provinces (Beijing, Shandong, Anhui, Jiangsu and Shanghai), three northwestern regions (Gansu, Xinjiang-Urumqi, and Korla), two middle-west provinces (Shaanxi and Ningxia), two western providence (Qinghai and Tibet), four southwestern regions (Sichuan, Yunnan-Kunming, Lincang, and Guizhou), three southeastern provinces (Fujian, Guangdong, and Guangxi), and five central regions (Henan, Hebei, Shanxi-Yuncheng, Datong, and Hubei) (Fig. 2).

\section{Prevalence of c.919-2A $>$ G mutation in different regions of China}

The SLC26A4 c.919-2A > G mutation was highly prevalent among 3271 deaf subjects from 27 regions of China (Table 1). The total number of subjects with SNHL carrying at least one c.919-2A $>$ G minor allele was 408 (12.5\%). The detection rate ranges broadly from near $28 \%$ to below $1 \%$ based on ethnicity. The allele frequency is higher than $12 \%$ in eight regions and lower than $4 \%$ in five regions, yielding a nationwide average frequency of $8.7 \%$.

c.919-2A $>$ G homozygote, heterozygote, and allele frequencies varied widely across different regions of China. Among the 3271 subjects, 158 (4.8\%) were homozygous and $250(7.6 \%)$ were heterozygous for the c.919-2A $>\mathrm{G}$ mutation (Table 1). The frequency of homozygous c.919-2A $>\mathrm{G}$ varied from $0 \%$ in Lhasa, Tibet, Liuzhou, and Guangxi, to $12.2 \%$ in Anyang, Henan. Heterozygosity for c.919-2A $>$ G varied from $0.9 \%$ in Lhasa, Tibet, to $17.6 \%$ in Zhuozhou Gaobeidian, and Hebei. Four of eight cities where the c.919-2A $>$ G allele frequency was high (11.6-20.6\%) were located in the eastern provinces, and the remaining four cities were located in central provinces adjacent to the eastern coast (Fig. 2). The proportion of c.919-2A $>\mathrm{G}$ homozygotes and heterozygotes was approximately equal in nearly half the regions, whereas the other half showed twice as many c.919-2A $>$ G heterozygotes versus homozygotes.

\section{Variable frequency of the c.919-2A $>G$ mutation across ethnic groups in China}

Regional variability in c.919-2A $>$ G MAF is at least partly because of prevalence of different ethnic groups in the regions studied. The total populations of Han, Tibetan, Hui, Man, Mon, Uigur, and Southwestern ethnic groups in China are 1137.4, 5.4, 9.8, 10.7, 5.8, 8.4, and 57.1 million, respectively (http://www. cnmuseum.com/intro/renkou_intro.asp; accessed August 14, 2007).

The distribution of c.919-2A $>\mathrm{G}$ homozygotes and heterozygotes differs among Chinese ethnic groups. The percentage of subjects carrying homozygous and heterozygous c.919$2 \mathrm{~A}>\mathrm{G}$ are $9.4 \%$ and $6.3 \%$ in Mon groups, $5.3 \%$ and $8.5 \%$ in Han groups, $4.1 \%$ and $5.0 \%$ in Hui groups, and $0.8 \%$ and $2.3 \%$ in the southwestern group.

Among ethnic groups in the southwest and northwest regions of China, the c.919-2A $>$ G mutation occurs at a much lower frequency compared with Han and Mon groups (45-fold difference). MAF for c.919-2A > G among Mon, Han, Hui, Southwestern ethnic groups, Uigur, and Tibetan was $0.125,0.096,0.066,0.019,0.000$, and 0.000 , respectively (Table 2). 


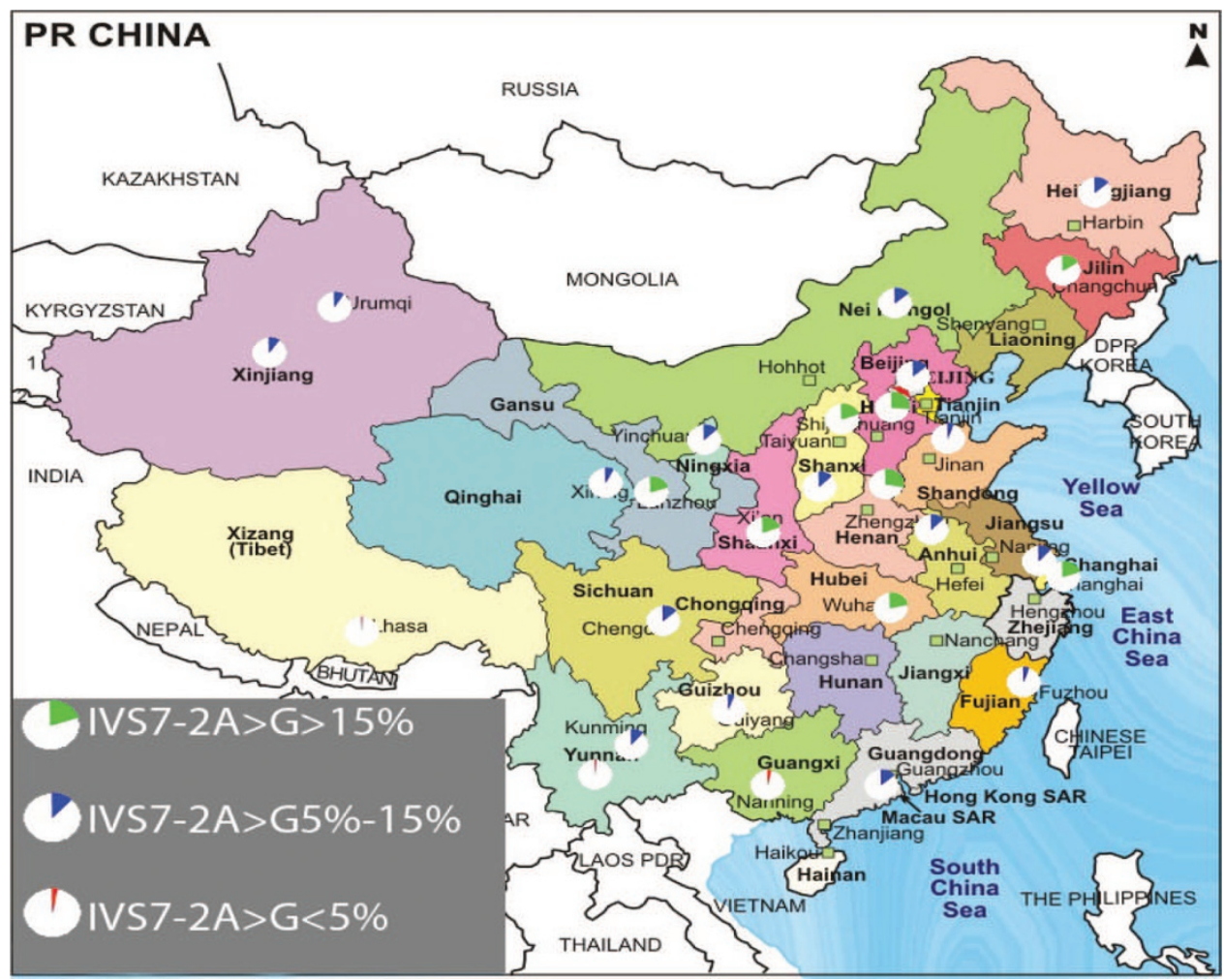

Fig. 2. Geographic distribution and the proportion of subjects carrying at least one c.919-2A $>\mathrm{G}$ minor allele in each region studied.

The higher proportion of homozygotes for c.919-2A $>$ G in Mon and Han suggested a founder effect from a common ancestor, which is supported by the fact that Mon and Han are living together in many places with long history. These results also suggest that full gene sequencing is necessary to establish the mutation spectrum and mutation frequency within Chinese minority ethnic populations.

We found a statistically significant difference in c.919$2 \mathrm{~A}>\mathrm{G}$ MAF among these representative ethnic groups (Table 3 ). We performed a pairwise comparison of MAF using a corrected $P$ value of approximately 0.00313 as a significance cutoff. Differences in MAF between the Mon, Han, Hui, Southwestern ethnic groups, Uigur, and Tibetan were statistically significant. Mon Chinese had the highest c.919-2A $>$ G MAF (0.125), which is statistically significant when compared with minorities in the southwest and northwest $(P<0.001)$.

\section{Imaging and sequencing to confirm the presence of EVA}

To determine how EVA is concordant with the homozygotes or heterozygotes, and how many of the heterozygotes may be coincidental occurrence as a carrier, we selected 38 subjects with homozygous and 30 subjects with heterozygous c.919-2A $>G$ mutation for temporal bone CT scan. All the homozygotes had EVA; 24 of 30 heterozygotes had EVA; and the remaining six heterozygotes had no EVA. This result is consistent with another study that we have done (Dai et al., unpublished data), which

Table 3

Pairwise comparison of SLC26A4 c.919-2A > G allele frequency among different ethnic groups in China

\begin{tabular}{|c|c|c|c|c|c|}
\hline & \multicolumn{5}{|c|}{$P$} \\
\hline & $\begin{array}{c}\text { Mongol } \\
(8 / 64,12.5 \%)^{a}\end{array}$ & $\begin{array}{c}\text { Han } \\
(533 / 5566,9.6 \%)\end{array}$ & $\begin{array}{c}\text { Hui } \\
(16 / 242,6.6 \%)\end{array}$ & $\begin{array}{c}\text { Southwest ethnic groups } \\
(5 / 262,1.9 \%)\end{array}$ & $\begin{array}{l}\text { Uigur } \\
(0 / 130,0)\end{array}$ \\
\hline $\operatorname{Han}(533 / 5566,9.6 \%)$ & 0.394 & & & & \\
\hline Hui $(16 / 242,6.6 \%)$ & 0.123 & 0.144 & & & \\
\hline $\begin{array}{l}\text { Southwest ethnic groups } \\
\quad(5 / 262,1.9 \%)\end{array}$ & 0.001 & 0.000 & 0.012 & & \\
\hline Uigur $(0 / 130,0)$ & 0.000 & 0.000 & 0.001 & 0.166 & \\
\hline Tibetan $(0 / 232,0)$ & 0.000 & 0.000 & 0.000 & 0.063 & 1.000 \\
\hline
\end{tabular}

${ }^{a}$ Number of c.919-2A $>$ G alleles/number of total alleles = \% allelic frequency; the corrected significance level is 0.00313. The populations of Mongol, Han, Hui, Southwestern ethnic groups, Tibetan, and Uigur are 5.8, 1137.4, 9.8, 57.1, 5.4, and 8.4 million, respectively (http://www.cnmuseum.com/intro/renkou_intro.asp). 
screened 131 children with bilateral severe to profound SNHL for c.919-2A $>\mathrm{G}$ before CT evaluation and found that all of the homozygotes and $80 \%$ of the heterozygotes have EVA confirmed by a follow-up CT imaging. Thus, these data suggest that approximately $20 \%$ individuals with heterozygous c.919-2A $>$ G mutation in this cohort are carriers.

In addition, 18 children with bilateral severe to profound SNHL and EVA were found to have one c.919-2A $>$ G mutation. Of them, a second pathogenic mutation was detected in eight children followed by a direct sequencing for entire coding region (Dai et al., unpublished data).

\section{Frequency of the c.919-2A $>\mathbf{G}$ mutation in Chinese controls}

We also examined the population prevalence of c.919A $>\mathrm{G}$ among normal hearing control subjects from the Han and Uigur. Among 185 normal hearing controls from the Han of northern and southern areas of China, we identified four individuals with the heterozygous c.919-2A $>$ G mutation. Among 152 normal hearing controls from the Uigur of Xinjiang we identified no individuals with the heterozygous c.919-2A $>\mathrm{G}$ mutation. Thus, the percentage of c.919-2A $>\mathrm{G}$ carriers in the Han population is approximately $2.2 \%$, whereas ethnic minority groups may show much lower carrier rates.

\section{DISCUSSION}

This study analyzed a total of 6542 SLC26A4 alleles, representing the largest study of the common SLC26A4 mutation, c.919-2A $>\mathrm{G}$, in Chinese subjects with moderate to profound SNHL. An estimated 2 million people in China have inherited bilateral SNHL. ${ }^{25}$ Our results suggest that the c.919-2A $>\mathrm{G}$ mutation would be present in approximately $12.5 \%$ of Chinese children with bilateral moderate to profound SNHL, translating to a positive test result in approximately 250,000 Chinese individuals with hearing loss.

These findings facilitate implementation of genetic testing by defining the prevalence of c.919-2A $>\mathrm{G}$ within a large and diverse population. Modern China is a melting pot of people of more than 56 different ethnic backgrounds, all considered Chinese by heritage. The regional variability in c.919-2A $>\mathrm{G}$ allele frequencies correlates with the geographic distribution of ethnic groups. Increasingly, minority groups such as Man from the northeast, Zang and Hui from the western, Mon from Mongolia, and aborigines from the southwest part are admixing with Hans. Consequently, homozygote frequency can be expected to transiently decline according to Wahlund principles. ${ }^{26,27}$ Analysis of controls from various regions and populations of China to determine the accurate carrier rate was not performed here, but would be expected to correlate with the mutation prevalence among cases.

A rational approach to genetic testing for SNHL includes consideration of methodology. PCR/restriction-fragment-length polymorphism is relatively straightforward, rapid and inexpensive, enabling this genetic service to be readily available to Chinese individuals with SNHL and their family members. For the sake of comparison, we estimate that $\mathrm{PCR} /$ restriction-fragment-length polymorphism screening of c.919-2A $>\mathrm{G}$ would cost 18.0 Chinese Yuan (RMB) per sample, equivalent to approximately US $\$ 2.50$. This could be accomplished for approximately half the cost of Universal Newborn Hearing Screening in China. Detection of heterozygous c.919-2A $>$ G should prompt screening of the entire SLC26A4 gene for the second mutant allele. Our results also suggest that full gene sequencing is necessary to establish the mutation spectrum and mutation frequency within Chinese minority ethnic populations.

Clinical sensitivity for targeted mutation analysis of c.919$2 \mathrm{~A}>\mathrm{G}$ among Chinese subjects with SNHL would be higher if performed only on individuals with documented EVA. c.919$2 \mathrm{~A}>\mathrm{G}$ is the most common SLC26A4 mutation associated with EVA among the Han Chinese and Korean populations, with allele frequencies of 0.63 among deaf Chinese with EVA in Taiwan ${ }^{20}$ and 0.17 among deaf Koreans with EVA. ${ }^{19}$ With no information for EVA, Chinese subjects with SNHL as a group still seem to have the highest c.919-2A $>$ G MAF among Asian populations: 0.087 in this study compared with 0.022 in Koreans where this variant is considered common, accounting for $36 \%$ of SLC26A4 alleles among Korean probands with SNHL in one study. ${ }^{17}$

Other genes, such as $G J B 2$ and $G J B 6$, also play a role in many cases of SNHL. The lack of an identified mutation in the majority of the subjects with SNHL in China underscores the potential benefits of comprehensive genetic testing based on initial screening for c.235delC in $G J B 2$ and c.919-2A>G in SLC26A4, the two most common mutations among Chinese with SNHL as demonstrated by our previous study ${ }^{28}$ and this report. Such an approach would lead to improved recurrence risk counseling and appropriate clinical follow-up.

Genetic testing is now available for a number of genes related to hearing loss, but the most efficient utilization of resources for genetic testing will follow from studies that determine the most prevalent mutations in the population to be tested. Further studies of mutation prevalence and clinical phenotyping are essential to providing cost effective access to diagnostic testing.

\section{ACKNOWLEDGMENTS}

This work was supported by the Chinese National Nature Science Foundation Research Grant 30572015, Beijing Nature Science Foundation Research Grant 7062062 (to P.D.), and Chinese Capital Medical Development Scientific Funding 2005-1032 (to D.H.). Dr. Bai-Lin Wu received support from the MDA Foundation and NIH-CETT Program to develop cost-effective assays for genetic tests.

The authors thank the referring physicians and parents for their support and cooperation.

\section{References}

1. Cohen MM, Gorlin RJ. Epidemiology, etiology and genetic patterns. In: Gorlin RJ, Toriello HV, Cohen MM, editors. Hereditary hearing loss and its syndromes. Oxford: Oxford University Press, 1995:9-21.

2. Bitner-Glindzicz M. Hereditary deafness and phenotyping in humans. Br Med Bull 2002;63:73-94. 


\section{Dai et al.}

3. Li XC, Everett LA, Lalwani AK, et al. A mutation in PDS causes non-syndromic recessive deafness. Nat Genet 1998;18:215-217.

4. Everett LA, Glaser B, Beck JC, et al. Pendred syndrome is caused by mutations in a putative sulphate transporter gene (PDS). Nat Genet 1997;17:411-222.

5. Usami S, Abe S, Weston MD, Shinkawa H, Van Camp G, Kimberling WJ. Nonsyndromic hearing loss associated with enlarged vestibular aqueduct is caused by PDS mutations. Hum Genet 1999;104:188-192.

6. Morton CC, Nance WE. Newborn hearing screening-a silent revolution. N Engl J Med 2006;354:2151-2164.

7. Reardon W, Coffey R, Phelps PD, et al. Pendred syndrome-100 years of underascertainment? QJM 1997;90:443-447.

8. Lasak JM, Welling DB. The enlarged vestibular aqueduct syndrome. Curr Opin Otolaryngol Head Neck Surg 2000;8:380-383.

9. Preciado DA, Lawson L, Madden C, et al. Improved diagnostic effectiveness with a sequential diagnostic paradigm in idiopathic pediatric sensorineural hearing loss. Otol Neurotol 2005;26:610-615.

10. Chang E, Kölin KA, Nishimura C, Fischer S, Smith RJH. Pendred/BOR homepage: SLC26A4 mutation database. 2008. Available at: http://www.healthcare.uiowa.edu/ labs/pendredandbor/.

11. Campbell C, Cucci RA, Prasad S, et al. Pendred syndrome, DFNB4, and PDS/ SLC26A4 identification of eight novel mutations and possible genotype-phenotype correlations. Hum Mutat 2001;17:403-411.

12. GeneReviews: Pendred syndrome/DFNB4. 2007. Available at: http://www.genetests. org/. Accessed August 14, 2007.

13. Coyle B, Reardon W, Herbrick JA, et al. Molecular analysis of the PDS gene in Pendred syndrome. Hum Mol Genet 1998;7:1105-1112.

14. van Hauwe P, Everett LA, Coucke P, et al. Two frequent missense mutations in Pendred syndrome. Hum Mol Genet 1998;7:1099-1104.

15. Gonzalez Trevino O, Karamanoglu Arseven O, Ceballos CJ, et al. Clinical and molecular analysis of three Mexican families with Pendred's syndrome. Eur J Endocrinol 2001;144:585-593.

16. Lopez-Bigas N, Melchionda S, de Cid R, et al. Identification of five new mutations of PDS/SLC26A4 in Mediterranean families with hearing impairment. Hum Mutat 2001;18:548.
17. Park HJ, Shaukat S, Liu XZ, et al. Origins and frequencies of SLC26A4 (PDS) mutations in east and south Asians: global implications for the epidemiology of deafness. J Med Genet 2003;40:242-248.

18. Tsukamoto K, Suzuki H, Harada D, Namba A, Abe S, Usami S. Distribution and frequencies of PDS (SLC26A4) mutations in Pendred syndrome and nonsyndromic hearing loss associated with enlarged vestibular aqueduct: a unique spectrum of mutations in Japanese. Eur J Hum Genet 2003;11:916-922.

19. Park HJ, Lee SJ, Jin HS, et al. Genetic basis of hearing loss associated with enlarged vestibular aqueducts in Koreans. Clin Genet 2005;67:160-165.

20. Wu CC, Yeh TH, Chen PJ, Hsu CJ. Prevalent SLC26A4 mutations in patients with enlarged vestibular aqueduct and/or Mondini dysplasia: a unique spectrum of mutations in Taiwan, including a frequent founder mutation. Laryngoscope 2005;115: 1060-1064.

21. Hutchin T, Coy NN, Conlon $\mathrm{H}$, et al. Assessment of the genetic causes of recessive childhood non-syndromic deafness in the UK-implications for genetic testing. Clin Genet 2005;68:506-512.

22. Coucke PJ, van Hauwe P, Everett LA, et al. Identification of two different mutation in the PDS gene in an inbred family with Pendred syndrome. J Med Genet 1999;36: 475-477.

23. Blons H, Feldmann D, Duval V, et al. Screening of SLC26A4 (PDS) gene in Pendred's syndrome: a large spectrum of mutations in France and phenotypic heterogeneity. Clin Genet 2004;66:333-340.

24. Yang JJ, Tsai CC, Hsu HM, Shiao JY, Su CC, Li SY. Hearing loss associated with enlarged vestibular aqueduct and Mondini dysplasia is caused by splice-site mutation in the PDS gene. Hear Res 2005;199:22-30.

25. Dai P, Liu X, Yu F, Zhu Q. Molecular etiology of patients with nonsyndromic hearing loss from deaf-mute schools in 18 provinces of China. Chin J Otol 2006;4:1-5.

26. Wahlund S. Zuzammensetzung von Populationen und Korrelationser-scheinungen vom Standpunkt der Vererbungslehre aus betrachtet. Hereditas 1928;11:65-106.

27. Ding YC, Wooding S, Harpending HC, et al. Population structure and history in East Asia. Proc Natl Acad Sci USA 2000;97:14003-14006.

28. Dai P, Yu F, Han B, et al. The prevalence of the 235delC GJB2 mutation in a Chinese deaf population. Genet Med 2007;9:283-289. 